\title{
RECONSTRUCCIONES URETERALES TOTALES EN CIRUGIA EXPERIMENTAL
}

\author{
Doctores Gustavo Escallón Caycedo y Manuel Augusto Rodríguez-Gtz.
}

\begin{abstract}
La cirugía no empieza con la intervención y termina con la mejoría. Se pierde y confunde con la historia clínica y sólo es justipreciable cuando habla el histopatólogo y la interpreta la estadística. IX. A. BONILLA NAAR. Decálogo del Cirujano.
\end{abstract}

\section{INTRODUCCION}

Uno de los problemas más frecuentes ante los que se encuentra abocado el cirujano en operaciones pélvicas o de vías urinarias altas es la posibilidad de la sección accidental o intencional del uréter, seguida de su necesaria reconstrucción plástica. Esta reconstrucción no siempre da resultados felices en la práctica.

Es, pues, indispensable pensar en el desarrollo de una técnica quirúrgica que permita la restauración adecuada de la continuidad de la luz ureteral y la prevención de fístulas o estenosis tan frecuentes en estos pacientes.

Debemos suponer la existencia de métodos y medios quirúrgicos que hagan posible la regeneración total del uréter por sí mismo y sin necesidad de emplear anastomosis o reemplazos con materiales naturales o artificiales como los descritos hasta el presente.

Aprovechando la propiedad de regeneración orgánica de los tejidos, conseguimos que el uréter continúe con sus funciones fisiológicas; interrumpidas por causas patológicas, congénitas o adquiridas.

En diciembre de 1958 se inició el trabajo que a continuación se presenta, advirtiendo que sus bases son estrictamente experimentales por el momento y que sus resultados, de interés eminentemente práctico, tienen enfoque humano, que quizás en un nuevo trabajo lograremos comprobar. 
Los Departamentos Científico y Administrativo del Laboratorio Cup, de Bogotá, fueron estímulo para realizar esta obra.

Nuestro agradecimiento también para el Departamento de Anatomía Patológica del Instituto Nacional de Cancerología, por los estudios histológicos de las piezas quirúrgicas. Igual cosa, a todas aquellas personas que en una u otra forma contribuyeron a la realización de estas experiencias.

Intencionalmente omitimos métodos quirúrgicos y resultados obtenidos experimentalmente por diferentes investigadores, después de hemi-resecciones parciales o reemplazos de uréter por otros materiales.

Se mencionan sí en la bibliografía, para consultas y confrontaciones particulares.

El lector podrá enterarse, más adelante, de cuáles fueron nuestros propósitos al pretender y obtener por primera vez la regeneración de un segmento ureteral total después de su resección experimental, trabajando en un volumen de diecinueve perros.

\section{TECNICAS QUIRURGICAS}

Métodos de anestesia.-Incisión._Vía de acceso.-Maniobras sobre uréter. Material plástico usado.-Modo de fijación del polietileno a los extremos. Post-operatorio.-Exámenes complementarios: Rayos $X$.

No se empleó premedicación. Inducción con Tiopental sódico, inyec- tando $0.0125 \mathrm{gm}$. I. V. por kilo de peso. Intubación orotraqueal (cánula semi-rígida). Mezcla éter-oxígeno. Este procedimiento fue el usado en todos los casos. En los cuatro primeros perros se utilizó la incisión lumbar derecha tratando de abordar el riñón y el uréter derechos por vía extraperitoneal en una forma similar, a la que se practica en los humanos.

Se encontró gran dificultad en hacer el rechazo del peritoneó, debido a la gran fragilidad de esta estructura. Prácticamente en todos los casos de operación fue necesario hacerla transperitoneal a pesar de la incisión lumbar efectuada.

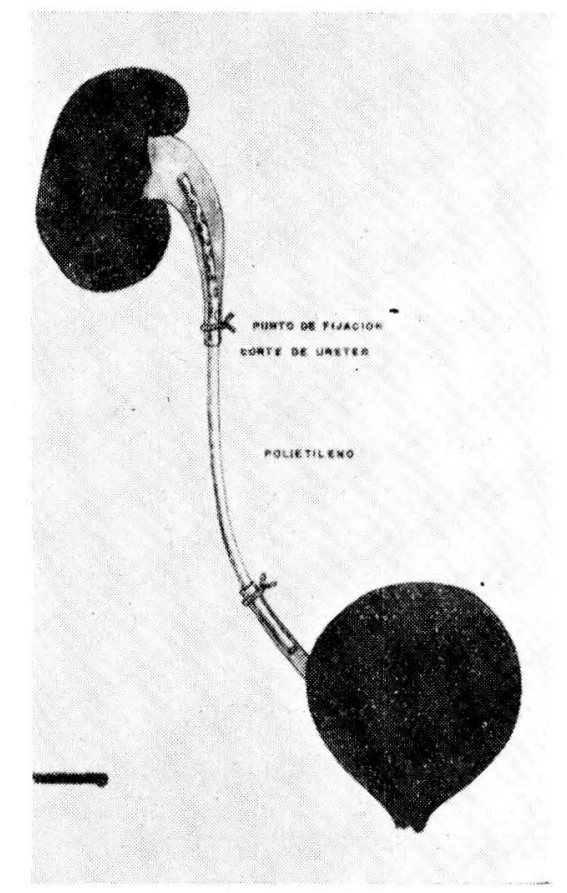

Figura 1. Polietileno desde pelvis renal, hasta tercio inferior de uréter. Punto de fijación anudado sobre cada extremo ureteral, para sostener el tubo de Polietileno. 
Como se trata de comprobar la posibilidad de regeneración de un segmento ureteral de aproximadamente cinco a diez centímetros ( 5 a 10 cms.) de longitud, se procede a disecar para luego resecar el mencionado segmento. Acto seguido se coloca un tubo de Polietileno desde la pelvis renal hasta la porción inferior o distal del uréter o hasta la vejiga con el objeto de continuar el fiujo urinario. El calibre de estos tubos osciló entre PE-240 y PE-205 Ref. según cada caso.

En los cuatro primeros perros operados se efectuó la fijación del tubo de Polietileno por medio de un nudo circular en la parte superior y en la parte inferior del uréter. Es decir, en los dos extremos que quedan de la resección previamente hecha, con el objeto de evitar la extravasación de orina, como se ilustra en la figura 1.

Suturas absorbibles y no absorbibles fueron empleadas en el cierre en tres planos de todos los animales. Durante los dos primeros días de post-operatorio los animales estuvieron bajo vigilancia de personal encargado.

El post-operatorio ordenado para todos los casos fue el siguiente: Penicilina, analgésicos, antiespasmódicos; nada por boca en los dos primeros días. Posteriormente: Proteínas, minerales, alimentación adecuada normal.

Las placas de Rayos X (Urografía I. V.) fueron tomadas teniendo el animal colocado el catéter de Polietileno en el uréter, y después de su extracción. Se utilizó el método siguiente:
Animal en ayunas desde el día anterior.

Tiopental sódico inyectando 0.0075 Gm. por vía I. V. y por kilo de peso. Así se obtuvo quietud y buena respiración para la toma de placas en espiración. Acto seguido inyectamos por I. V.: 2 Gm. de Sal sódica y 13,2 Gm. de Sal metil-glutamínica del Acido 3.5 diacetilamino 2.4.6. triyodobenzoico (Urografina al $76 \%$ ).

La placa tomada a uno de los cinco primeros animales se encuentra ilustrada en la fig. 2.

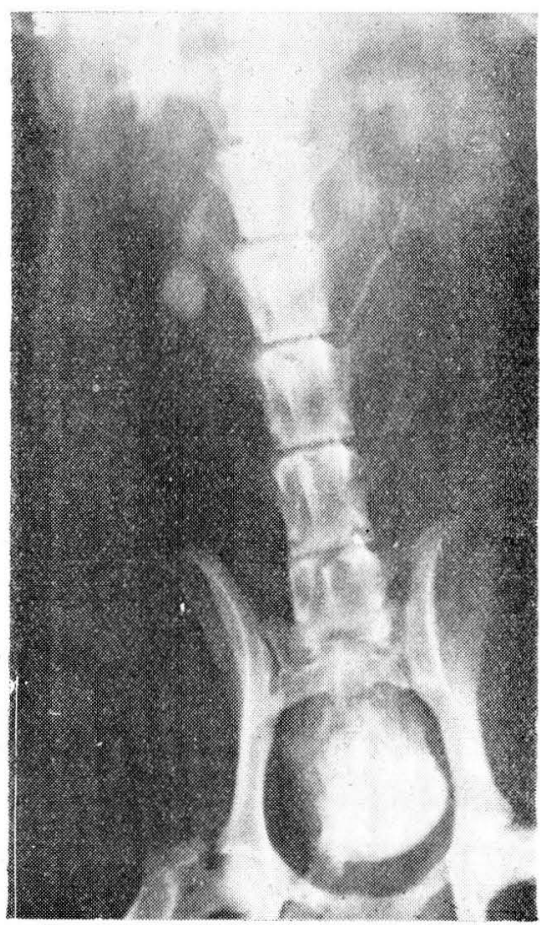

Figura 2. Se observa dilatación de pelvis renal derecha. Uréter con estenosis producida por fibrosis, hacia la unión del tercio superior con el tercio medio. 


\section{VARIANTES A LAS PRIMERAS INTERVENCIONES}

En reunión previa resolvimos abordar el animal por incisión mediana (infraumbilical). Habíamos deducido ya por las operaciones anteriores, que la vía transperitoneal era la más adecuada para efectuar la operación que nos ocupa. Y la línea media nos brinda la oportunidad de intervenir sobre el lado opuesto dado el caso de encontrar alguna anomalía sobre el derecho, que era el convenido para todos los casos de este trabajo.

Comentario: Los cinco primeros casos nos sirvieron en realidad para sacar conclusiones que se aplicarían a las demás experiencias y nos llevarían a poder presentar una casuística favorable.

Estas conclusiones son las siguientes: 1ạ Usar en adelante la vía mediana infraumbilical.

2a Continuar con el uso de la férula de Polietileno para permitir la reconstrucción del segmento resecado.

3a Modificar el método de fijación del tubo de Polietileno a los extremos del uréter, pues el usado hasta el presente, si bien permite temporalmente el paso de orina, no ofrece garantías de ninguna clase por su expulsión a los pocos días.

Además, un nudo circular (fig. 1), impide completamente la circulación de los extremos ureterales y, de este modo, es imposible esperar reconstrucción a expenșas de un segmento que padece de isquemia total o parcial.
Rev. Col. Obs. y Ginec.

Por ocurrencia de uno de nosotros (MARS), se resolvió que debería hacerse la fijación del tubo de Polietileno, por medio de un punto de seda perforante que atravesando las luces de uréter y Polietileno y anudado flojamente a modo de "punto para fijar dren", permita la adaptación uréter-Polietileno, evitando la expulsión del tubo, la retracción de los extremos del uréter seccionado y no impida la circulación de dichos extremos, como se ilustra en la figura 3 .

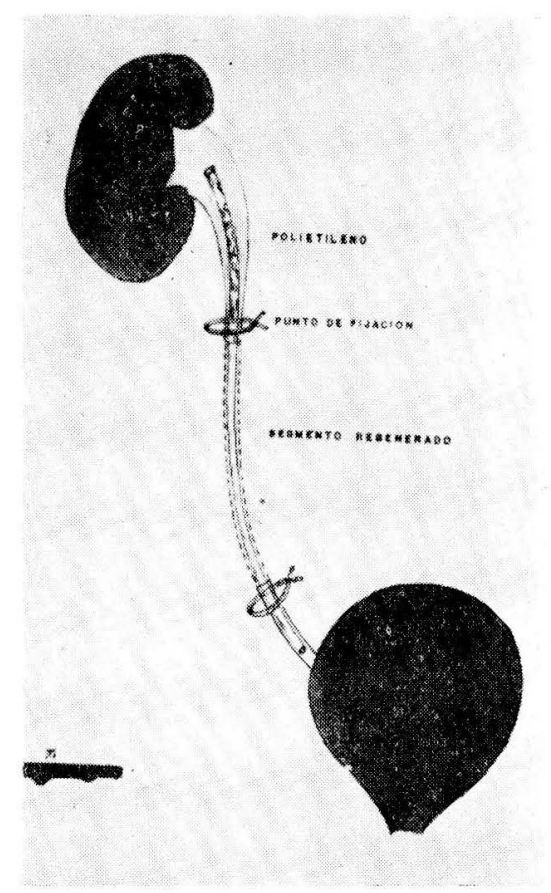

Figura 3. Polietileno desde pelvis renal, hasta tercio inferior de uréter. Punto de fijación anudado flojamente a modo de "punto para fijar dren", con el objeto de permitir la adaptación uréter-Polietileno, evitando la expulsión del tubo, la retracción de los cabos de sección $y$ no impedir la circulación de los extremos uretrales. 
Utilizando las modificaciones anteriores: Incisión mediana, acceso transperitoneal a las vías urinarias, misma técnica quirúrgica inicial, "punto para fijar dren", se obtuvieron los resultados radiográficos, de laboratorio, macroscópicos e histológicos que observaremos más adelante. Traemos a cuento algunas historias clínicas ilustradas.

PERRO número 15.

Sexo: Macho. Peso: 18 kgms.

En diciembre 10 de 1959, operación. Técnica descrita. Con instrumento cortante apertura de peritoneo parietal posterior. Resección de $5 \mathrm{cms}$. de uréter derecho. Colocación del Polietileno y fijación con seda. Sutura del peritoneo parietal posterior. Dren contralateral. Cierre por planos.

En abril 8 de 1960, urografía I. V.: Fig. 4.

En mayo 12 de 1960, segunda operación. Dilatación moderada de la pelvis

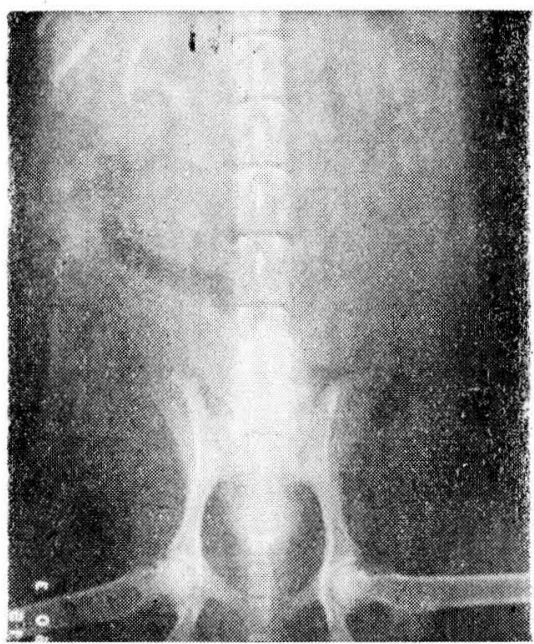

Figura 4. Morfología renal derecha normal.

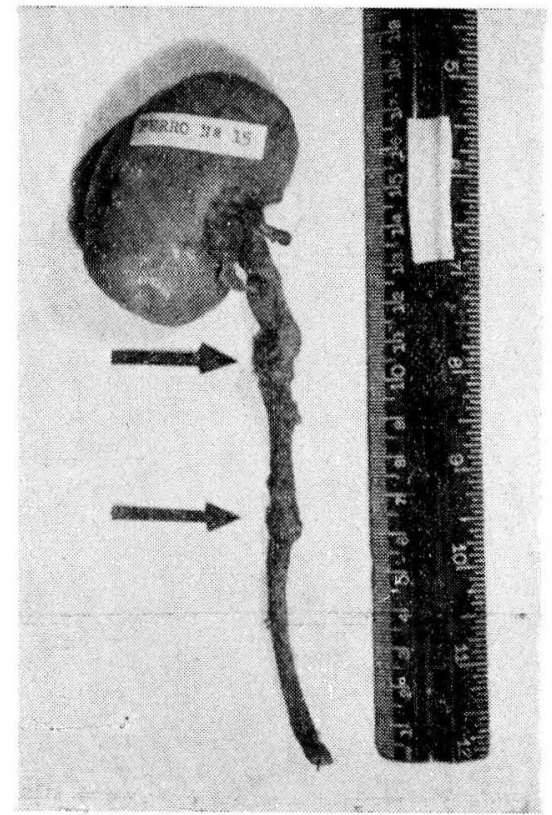

Figura 5. Riñón, pelvis y uréter derechos de tamaño normal. Arteria $y$ venas renales conservadas post-urétero-nefrectomía derecha. Limitada por las flechas se observa la zona de regeneración en una extensión de 5 cms. aproximadamente. Uréter permeable en todo su trayecto.

renal. Extracción del Polietileno mediante ureterotomía media longitudinal por el segmento neoformado. $\mathrm{Al}$ practicar esta maniobra sobrevino desgarro del tercio medio reconstruído. Se solucionó suturando transversalmente el uréter con catgut crómico 00 . Muestra de orina y el material plástico de intubación se remitieron al laboratorio para estudio.

\section{RESULTADOS DEL EXAMEN DE LABORATORIO}

ORINA: $\mathrm{pH} 6,5$.

CULTIVO DE ORINA: En cultivos directos e indirectos se encuentran 

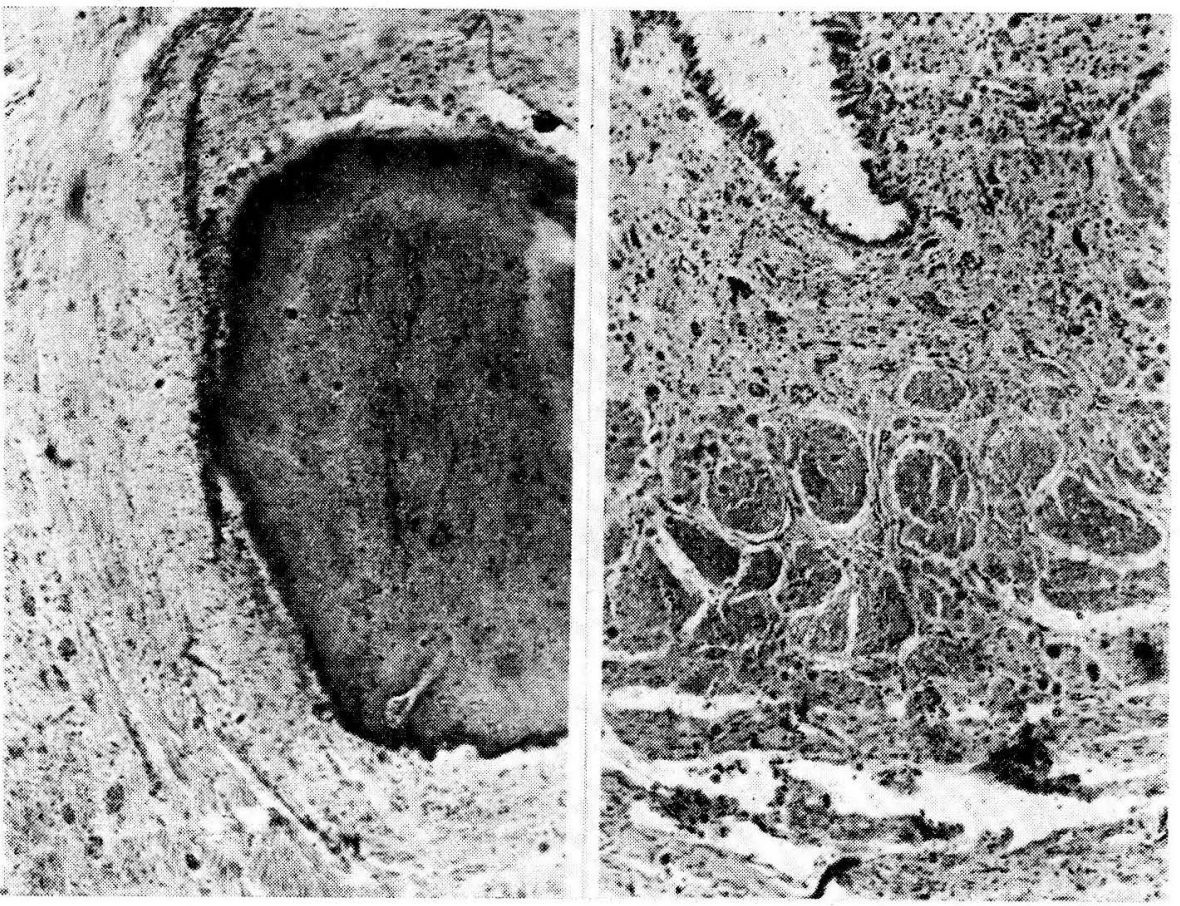

Figura 6. Lado derecho: Se encuentran las diversas capas del órgano: epitelial, muscular $y$ adventicial. Lado izquierdo: Entre las capas mucosas y muscular se observa metaplasia ósea, que no produce estenosis de la luz.

colonias de bacilos coli y estafilococos albus.

CULTIVO DEL POLIETILENO: En cultivos directos e indirectos no se observó crecimiento de bacterias. Los cultivos examinados durante 7 días fueron estériles.

En junio 30 de 1960, tercera operación. Totalmente reconstruído el uréter. Aparentemente no existen estrecheces a lo largo de su luz. No hay hidronefrosis. Discreta dilatación de pelvis renal. Nefrectomía y ureterectomía seccionándolo inmediatamente por encima de cruzar el canal deferente derecho. Fig. 5.
La pieza quirúrgica obtenida se remitió como todos los de esta experimentación, al Laboratorio de Anatomía Patológica.

Los cortes practicados a diferentes niveles del segmento neoformado mostraron (fig. 6).

PERRO número 16.

Sexo: Macho. Peso: 13 kgms.

En febrero 11 de 1960, misma técnica anterior. Resección de $6 \mathrm{cms}$. de uréter. Colocación del Polietileno. Fijación. Sutura del peritoneo parietal posterior. Dren contralateral. 
En mayo 18 de 1960. Urografía I. V.: Morfología renal derecha normal. Polietileno presente. No hay dilatación del uréter.

En mayo 19 de 1960, reoperación. Reconstrucción total del uréter en el segmento seccionado. Extracción del Polietileno. Como no fue posible extraer el tubo completamente, fue necesario practicar ureterotomía longitudinal, desde el tercio medio reconstruído hasta el tercio superior. Accidentalmente se desgarró el uréter con sección transversal

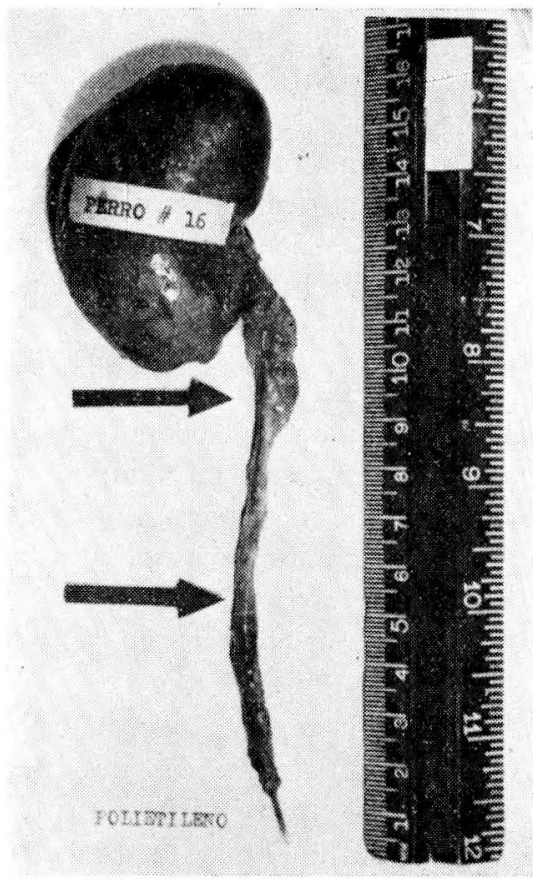

Figura 7. Riñón de tamaño y forma normales. El uréter es permeable en toda su longitud $y$ se encuentra canalizado por un tubo de Polietileno. La zona de regeneración señalada por las flechas, es de una extensión de $5 / \mathrm{cms}$. Dicha reconstrucción, como se ilustra en el grabado, se observó en 2 ocasiones en el mismo animal (ver texto). completa y pérdida de substancia. Se procedió entonces nuevamente a practicar urétero-plastia con la misma técnica usada en febrero 11 de 1960. Es decir, como si se tratara de la primera intervención. Un segmento de uréter desgarrado (1/3 medio reconstruído). Se envió a Anatomía Patológica para estudio.

Descripción microscópica: Los cortes muestran uréter con erosión del epitelio. Apreciamos frecuentes capitulares neoformados y pequeños cúmulos inflamatorios. El uréter se presenta con todas sus túnicas, inclusive la muscular.

En julio 6 de 1960, tercera operación. Nuevamente reconstrucción total de uréter. No existen calcificaciones a lo largo de sus paredes. No hay hidronefrosis. Se procede a practicar uréteronefrectomía derecha, conservando el tubo de Polietileno dentro de la luz ureteral (fig. 7). La pieza quirúrgica se remite a Anatomía Patológica.

\section{INFORME DE ANATOMIA PATOLOGICA}

Descripción microscópica: Tercio medio (M) intervenido. Se encuentra mucosa, muscular y túnica adventicia. La adventicia está infiltrada por células inflamatorias, siendo frecuentes los polimorfonucleares (fig. 8).

\section{RESUMEN}

En un total de 19 perros de raza indefinida y de ambos sexos, cuyo peso osciló entre 10 y 18 kilogramos, se reali- 


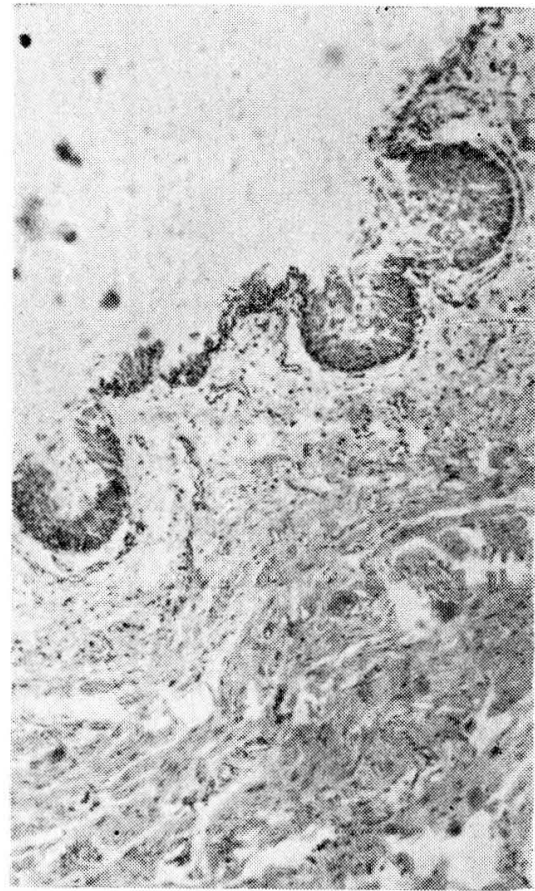

Figura 8. Tercio medio (M) intervenido. Se identifica uréter con todas sus túnicas normales, encontrándose completas las capas musculares. El corión está moderadamente infiltrado por células inflamatorias.

zaron intervenciones quirúrgicas destinadas a la resección de un segmento de uréter de aproximadamente 5 a $10 \mathrm{cms}$. de longitud y a su reconstrucción sobre tubo de Polietileno.

Todas las intervenciones se verificaron sin premedicación, usando para inducción el Tiopental sódico a la dosis de $0.0125 \mathrm{gm}$. por kilo de peso y por vía intravenosa, seguido de anestesia éteroxígeno a través de cánula endotraqueal.

La técnica quirúrgica incluyó incisiones lumbar o mediana infraumbilical y acceso transperitoneal al uréter.
Los cuidados post-operatorios incluyeron administración de antibióticos analgésicos y suplementos dietéticos.

Los animales fueron aislados e identificados cuidadosamente durante el post-operatorio.

Los controles radiográficos se hicieron después de cada intervención, teniendo el animal colocado el catéter de Polietileno en el uréter y después de su extracción. Se usó para los mismos medio opaco yodado por la vía I. V.

Se practicó además cultivo de laboratorio, de la orina y del material plástico usado, para comprobar tolerancia a la intubación ureteral. Todas las reconstrucciones ureterales fueron comprobadas histopatológicamente. Estudios completos tanto macroscópicos como microscópicos se encuentran en el Archivo del Instituto Nacional de Cancerología de Bogotá D. E.

Las piezas se obtuvieron después de la última intervención, cuando se sacrificó a los animales, o en tiempos variables después de la primera, en los casos en que hubo muerte post-operatoria.

\section{COMENTARIOS}

Se obtiene la regeneración de un segmento ureteral total, después de la resección de aproximadamente 5 a $10 \mathrm{cms}$. de su longitud. La técnica más adecuada hasta el presente para la reconstrucción de un segmento ureteral resecado, probablemente es la empleada por nosotros (fig. 2).

Existe muy buena tolerancia a la intubación ureteral con Polietileno, com- 
probada por los exámenes de laboratorio (fig. 9). En nuestros casos la regeneración de las 3 capas ureterales: Epitelial, muscular y adventicia, fue completa después de la resección total de un segmento determinado (fig. 10), aun en aquel animal en el cual la experiencia se hizo dos veces (fig. 8). La regeneración completa de las tres capas ureterales se confirma por las primeras urografías practicadas y por el primer control quirúrgico efectuado, así como por los estudios histológicos hechos a diferentes niveles sobre riñón y $3 / 3$ de uréter.

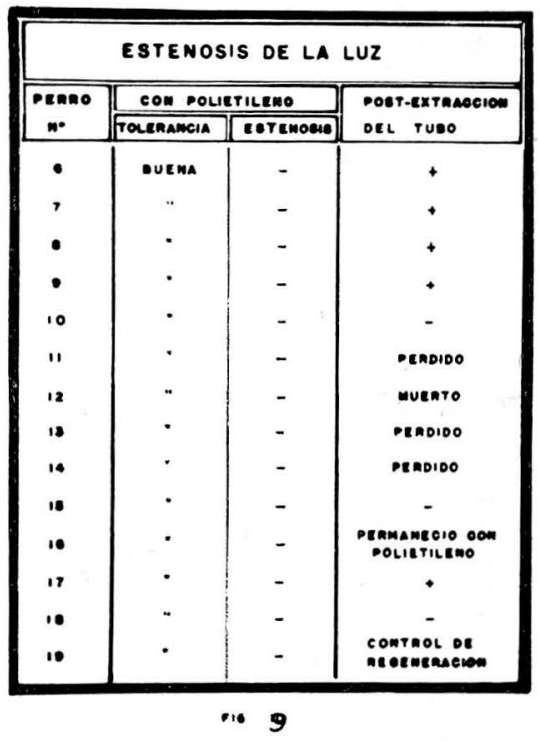

Figura 9.

Como tiempo promedio para la regeneración ureteral con todas sus capas, se necesitan de catorce a dieciséis semanas.

La metaplasia ósea que se presentó solamente en cuatro animales (fig. 10) se puede explicar, según la teoría de
Huggins (10), por influencia del epitelio de transición sobre ciertos fibroblastos convirtiéndolos en osteoblastos. De acuerdo con estas mismas experiencias de Huggins, este fenómeno es común en los perros, pero raro en el humano.

Naturalmente que en nuestra experiencia existe un factor físico irritante (seda).

Se llevarán a cabo estudios complementarios para tratar de suprimir este factor. Los animales que observamos con metaplasia ósea no presentaron obstrucción alguna y el flujo urinario fue normal mientras estuvieron cateterizados.

Como se puede observar, todos estos inconvenientes tienen más o menos solución si esta técnica se utilizara en el humano por las siguientes consideraciones:

1. Mejor paciente.

2. Permite colocación de drenes o sondas para drenaje urinario por pielostomía.

3. El lavado ureteral se puede hacer periódicamente con medios ácidos, con el fin de impedir la precipitación de sales de calcio. Estos dos puntos anteriores pueden realizarse combinando nuestra técnica, con la empleada por DAVIS (1-2-3).

4. Los exámenes complementarios como urografía y cistoscopia, dado el caso de que se quiera extraer el tubo de Polietileno por vía vesi$\mathrm{cal}$, para evitar una segunda operación, se pueden realizar perfectamente en el humano. 


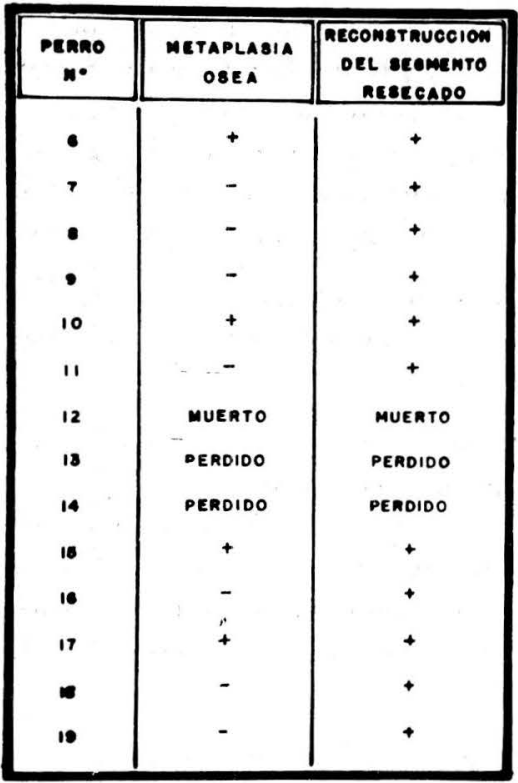

Figura 10.

Como resultado final, nuestra experiencia puede tener las siguientes aplicaciones médico-quirúrgicas en el ser humano:

1a En casos de estrecheces ureterales extensas, congénitas o adquiridas.

2a Por traumatismos que produzcan lesiones sobre el tracto ureteral.

3 a Por accidentes durante cualquier acto quirúrgico que hagan imposible otro tipo de intervención como reimplantación, etc. 4a Para evitar practicar otras vías de abocamiento ureteral, obviando las incomodidades ocasionadas al enfermo por ureterostomías cutáneas o urétero-enterostomías.

5ạ Lesiones por enfermedades neoplásicas: carcinomas o sarcomas, primitivos o metastásicos de órganos distantes o vecinos: cérvix que por invasión a parametrios produzcan estrecheces extensas, hidronefrosis, exclusión renal, etc.

6ạ Como método de corrección de intervenciones quirúrgicas sobre uréter que no hayan dado resultado satisfactorio.

7a Este trabajo puede ser base para ensayar técnicas quirúrgicas similares sobre órganos tubulares: Vías biliares, tracto urogenital del hombre y de la mujer, etc.

Naturalmente esta experiencia constituye solamente un paso más hacia el mejoramiento de la cirugía ureteral. Llegará el día en que se encuentren nuevos métodos que eviten por completo la metaplasia ósea, la estenosis y otros fenómenos agregados que se presentan ocasionalmente. Por nuestra parte continuaremos las investigaciones para poder contribuír en poco o mucho a la solución de estos problemas. 


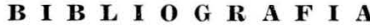

1. DAVIS, D. M.: Intubated Ureterotomy. Surg., Gyne., and Obst., ;6: 513-523, 1943.

2. DAVIS, D. M. : Intubated Ureterotomy. J. Urol., 59: 851-859, 1948.

3. DAVIS, D. M.: Intubated Ureterotomy. J. Urol., 66: 77-84, 1951.

4. HAM, F. C. and WEINBERG, S. R.: Experimental Studies of Regeneration of Ureter Without Intubación. J. Urol., 75: 43-51, 1956.

5. HINMAN Jr., F. and OPPENHEIMER, R. : Smooth Muscle Regeneration in Repair of Experimental Ureteral Defects. J. Urol., 75; 428-432, 1956.

6. HINMAN Jr., F. and OPPENHEIMER, R.: Ureteral Regeneration, IV, Fascial covering compared with fatty connective tissue. J. Urol., 76: 729-735, 1956.

7. HINMAN Jr., F. and OPPENHEIMER, R.: Ureteral Regeneration, VI, Delayed Urinary Flow in healing of unsplinted ureteral defects. J. Urol., 78: 138-144, 1957.

8. HINMAN Jr., F.: Ureteral Repair and the Splint. J. Urol., 78: 376-383, 1957.

9. HUFFMAN, W. L. McCORKLE, H. F. and PERSKY, L.: Ureteral regeneration following experimental segmental resection. J. Urol., 75: 796-800, 1956.

16. HUGGINS, C. B.: The formation of bone under the influence of epithelium of urinary tract. Arch., Surg., 22: 377-408, 1931.

11. RINKER, J. R.: Use of lock splint in intubated ureterotomy. J. Urol., 75: 52-54, 1956.

12. SISSON, GROSSMAN: Aparato urinario del perro. Anat., de los animales domésticos 606607, 1953.

13. ULM, A. H. and KRAUSS, L.: Total unilateral teflon ureteral substitutes in dog. J. Urol., 83: 575-582, 1960.

14. WEAVER, R. G.: Ureteral regeneration. J. Urol., 7 : : 164-172, 1957. 